\title{
A Auto-Eficácia e os Aspectos Comportamentais de Crianças com Dificuldade de Aprendizagem
}

\author{
Paula Cristina Medeiros ${ }^{12}$ \\ Sonia Regina Loureiro \\ Maria Beatriz Martins Linhares \\ Edna Maria Marturano \\ Universidade de São Paulo/Ribeirão Preto
}

\begin{abstract}
Resumo
O objetivo da pesquisa é avaliar as relações entre o desempenho acadêmico, o senso de auto-eficácia e os aspectos comportamentais, em 52 crianças de ambos os sexos, na faixa etária de oito anos a onze anos e onze meses, alunos de primeira a quarta série, com nível intelectual pelo menos médio inferior, divididas em: G1-26 crianças com queixa de dificuldade de aprendizagem, encaminhadas a um Ambulatório de Psicologia vinculado a um Hospital Escola; e G2-26 crianças com bom desempenho acadêmico avaliado por teste de Desempenho Escolar. Os instrumentos utilizados foram: Roteiro de Avaliação de Auto-eficácia e Escala Comportamental Infantil A2 de Rutter, além do teste de desempenho escolar. Observou-se que as crianças do G1 apresentaram uma avaliação significativamente mais baixa quanto ao senso de auto-eficácia e seus pais caracterizaram-nas com mais dificuldades comportamentais, comparativamente ao G2. O desempenho acadêmico mostrou-se relacionado ao senso de auto-eficácia e a indicadores de dificuldade comportamentais.
\end{abstract}

Palavras-chave: Dificuldade de aprendizagem; auto-eficácia; comportamento.

Self-Efficacy and Behavioral Aspects of Children with Learning Difficulties

Abstract

The aim of the present study was to assess the relationship among school performance, sense of self-efficacy, and behavioral aspects in 52 children of both sexes, aged from eight to 11 years and 11 months. The children were enrolled in the first to fourth grade and their intelligence level was assessed as of lower medium level at least. Subjects were divided in two groups: G1-26 children with learning difficulties referred to a Psychology Ambulatory of a School Hospital, and G2-26 children with good achievement, assessed by an academic performance test. The instruments used were: Self-Efficacy Assessment Form and Rutter Child Behavior Scale, as well as a school achievement test. G1 showed a significantly lower score for self-efficacy sense, compared to G2. A significant difference was also observed in the assessment made by parents, who characterized G1 children as having more behavioral difficulties. Academic performance was found to be related to self-efficacy sense and to indicators of behavioral problems.

Keywords: Learning difficulty; self-efficacy; behavior.

A experiência escolar pode contribuir para diferentes trajetórias de desenvolvimento, tendo impacto sobre as experiências futuras do indivíduo (Marturano, 1997). A época de ingresso na escolaridade formal coincide com a fase de desenvolvimento psicossocial, proposta por Erikson (1950/1971), que enfatiza o período entre os seis e doze anos como sendo a fase em que ocorre a crise evolutiva decorrente do desafio da produtividade versus inferioridade, onde a criança quer e precisa ser

${ }^{1}$ Endereço para correspondência: Rua Iguape, 603, apto. 62, Bairro Jardim Paulista, 14.090-090, Ribeirão Preto, SP.

${ }_{2}$ Este trabalho faz parte do projeto de mestrado da primeira autora, sob orientação da segunda, subvencionado pela FAPESP. reconhecida pela sua capacidade de realizar tarefas valorizadas no seu meio ambiente. $\mathrm{O}$ sucesso escolar, nesta fase, contribui para a resolução satisfatória desta crise, visto que garante à criança um desempenho valorizado pela sociedade (Lindahl, 1988; Marturano, 1997). Por outro lado, o insucesso acadêmico pode acarretar um senso de não cumprimento da sua tarefa psicossocial de desenvolvimento.

Os atrasos e problemas de aprendizagem foram durante muito tempo considerados como uma deficiência em determinada habilidade. No entanto, as teorias da deficiência apresentaram grandes dificuldades para estabelecer uma relação direta entre a dimensão psicológica atingida e o rendimento acadêmico 
(Boruchovitch, 1994; Martín \& Marchesi, 1995). Neste sentido, mais recentemente, tende-se a considerar a interação de uma série de fatores, cuja confluência específica determina o nível de rendimento da criança frente à situação de aprendizagem.

Ao se fazer referência às dificuldades de aprendizagem não se pode perder de vista a presença de distorções inerentes ao próprio sistema educacional e às influências ambientais que funcionam como contexto para as manifestações comportamentais e as peculiaridades do indivíduo que pode apresentar, no sistema escolar, o sintoma de não aprender (Linhares, 1998; Marturano, Linhares \& Parreira, 1993).

Estudos sobre o processo de ensino e aprendizagem vêm reforçando a importância das influências das variáveis internas como as escolhas, crenças, expectativas e afetos, que funcionam como mediadores da relação entre estímulo e resposta (Chapman, Cullen, Boersma \& Maguire, 1981). Dentre estas variáveis, destaca-se neste estudo a auto-eficácia. Define-se como auto-eficácia a crença do indivíduo sobre sua capacidade de desempenho em atividades específicas. Envolve o julgamento sobre suas capacidades para mobilizar recursos cognitivos e ações de controle sobre eventos e demandas do meio (Bandura, 1989a). Tais crenças influenciam as aspiraçõe e o envolvimento com metas estabelecidas, o nível de motivação, a perseverança face às dificuldades, a resiliência às adversidades, relacionando-se com a qualidade de pensamento analítico, a atribuição causal para sucesso e fracasso e a vulnerabilidade para o estresse e depressão. Os estudos em geral têm abordado como os fatores ambientais e pessoais afetam a auto-eficácia e como a auto-eficácia influencia a aprendizagem, motivação e realização acadêmica (Bandura, Barbaranelli, Caprara \& Pastorelli, 1996).

Os indivíduos possuem um sistema de crenças que afeta pensamentos, sentimentos e ações. Através da reflexão os indivíduos são capazes de se engajarem em auto-avaliações e alterarem seu próprio pensamento e comportamentos subseqüentes. Estas auto-avaliações incluem o senso de auto-eficácia (Bandura, 1989b; Schunk, 1995).

Segundo Schunk (1995), existem quatro principais formas de influências sobre o desenvolvimento do senso de auto-eficácia: a) experiências significativas, que são os resultados interpretados de um desempenho proposto, sendo que o sucesso aumenta o senso de auto-eficácia o fracasso o diminui; b) experiências através do outro, que são as observações sociais e a avaliação do desempenho dos outros, "se o outro pode fazer eu também posso"; c) persuasão social, que são as avaliações que as pessoas recebem dos outros; d) os estados psicológicos associados à emoções positivas ou negativas que influenciam na maneira como as situações são percebidas.

O constructo auto-eficácia tem sido particularmente aplicado às crianças que experienciam dificuldades de aprendizagem, afeto ou comportamento. A auto-eficácia dos estudantes, juntamente com outras crenças e atitudes para a aprendizagem, é forte preditor de desempenho acadêmico. A auto-eficácia influencia o desempenho acadêmico e, ao mesmo tempo, é influenciada por ele, tendo implicações no desenvolvimento da criança como um todo. Esta influência ocorre tanto por uma ação direta como através do seu impacto nos processos de motivação, auto-regulação e auto-percepção, nas expectativas de resultados, bem como escolhas e interesses, que por sua vez, afetam o nível e o tipo de comportamento (Bandura \& Schunk,1981; Pajares \& Valiante, 1997; Pintrich, Anderman \& Klobucar, 1994; Schunk, 1995).

Segundo Pajares (1996), pesquisas que abordam o senso de auto-eficácia dentro do contexto acadêmico sugerem que estudantes com alto senso de auto-eficácia são capazes de desempenhar tarefas acadêmicas usando mais estratégias cognitivas e metacognitivas e persistem por mais tempo do que aqueles com baixo senso. Em contrapartida, crianças que apresentam dificuldades de aprendizagem podem se julgar com baixo senso de autoeficácia quanto às suas capacidades de desempenharem com sucesso determinadas tarefas acadêmicas.

A dificuldade de aprendizagem quase sempre se apresenta associada a outros comprometimentos. Estudos têm revelado que comumente as crianças com dificuldades escolares manifestam paralelamente prejuízos de ordem emocional e comportamental (Graminha, 1994a). Linhares, Parreira, Maturano e Sant'ana (1993) observaram a presença de mais de uma área problema nos relatos dos pais que procuravam atendimento para seus filhos, havendo padrões de queixas combinadas. As queixas escolares apareciam associadas a problemas afetivos-sociais, relacionados ou não à escola, e a alterações de processos cognitivos.

A auto-eficácia exerce sua influência sobre o comportamento através de quatro processos que incluem: a cognição, motivação, afeto e seleção de atividades e ambientes (Bandura, 1993; Pajares, 1996; Schunk, 1995). Os efeitos do senso de auto-eficácia no processo cognitivo, recaem sobre o tipo de cenário antecipatório a ser construído. Pessoas com alto senso de auto-eficácia são capazes de visualizarem a si mesmos executando atividades com habilidades e apresentam boas construções 
cognitivas para resolverem problemas potenciais, possibilitando diretrizes positivas de desempenho. A crença na auto-eficácia, além de modificar regras de pensamento, determina o nivel de motivação. Quanto mais alto o senso de auto-eficácia, maior será o esforço despendido, a persistência, o envolvimento com metas e objetivos mais elevados. Pessoas duvidosas de suas capacidades enfraquecem seus esforços ou abandonam prematuramente suas tentativas. O processo afetivo é também fortemente afetado pela crença na auto-eficácia, porque pode influenciar na quantidade de estresse e ansiedade que uma pessoa vai experimentar diante de determinadas atividades. A eficácia no processo de seleção pode interferir no curso da vida através da influência nas escolhas de atividade e ambientes, baseadas na sua percepção do que é seguro, atingível, desafiador ou recompensador. As pessoas tendem a evitar atividades e situações que elas acreditam exceder suas capacidades (Bandura, 1993; Schunk, 1995)

Neste sentido, o presente trabalho tem como objetivo avaliar as relações entre o desempenho acadêmico, o senso de auto-eficácia e aspectos comportamentais de crianças do Ensino Fundamental.

\section{Método}

\section{Participantes}

Foram avaliadas 52 crianças, de ambos os sexos, na faixa etária entre oito anos e 11 anos e 11 meses, alunos de primeira a quarta série de 20 escolas da rede pública do município de Ribeirão Preto, distribuídas em dois grupos:

Grupo 1 - G1: Composto por 26 crianças (16 meninos e 10 meninas) encaminhadas a um ambulatório de Psicologia vinculado a um hospital escola com queixa de dificuldade de aprendizagem.

Grupo 2- G2: Composto por 26 crianças com bom desempenho acadêmico que freqüentam um Centro de Atendimento Integral a Crianças e Adolescentes (CAIC)

Para a seleção dos 52 sujeitos foram inicialmente avaliadas 88 crianças, sendo 36 procedentes do ambulatório e 52 do CAIC. Foram excluídos de ambos os grupos 26 sujeitos que apresentaram desempenho abaixo do nível intelectual médio inferior na avaliação intelectual através das Matrizes Progressivas Coloridas de Raven - Escala Especial - MPC (normas brasileiras de Angelini, Alves, Custódio \& Duarte, 1987) e aqueles que apresentaram história de tratamento neurológico e/ou psicológico no último ano. Considerou-se como condição para inclusão no G2 o desempenho no Teste de Desempenho Escolar - TDE (normas brasileiras de Stein,
1994), nas áreas de leitura e escrita, compatível com sua faixa etária e/ou série escolar, e a avaliação da professora através do Questionário para Caracterização do Desempenho e do Comportamento da Criança no Ambiente Escolar - DCCE (Machado, Figueiredo \& Selegato, 1989), indicando bom desempenho.

Na composição do G2 tomou-se como referência as crianças do G1, buscando-se o balanceamento com relação a sexo, idade da criança e nível de escolaridade dos pais. Após a seleção dos sujeitos, procedeu-se à comparação dos grupos através do teste não-paramétrico de Mann-Whitney, quanto às variáveis idade (G1/ $M=109,31$ e $\mathrm{G} 2 / M=110,42)$, escolaridade das mães (G1/M=6,27 e G2/M=6,81), escolaridade dos pais (G1/ $M=4,62$ e $G 2 / M=5,50)$, e não foram observadas diferenças estatisticamente significativas na comparação destas variáveis. Quanto ao nível intelectual das crianças ( $\mathrm{G} 1 / M=44,81$ e $\mathrm{G} 2 / M=57,88)$, observou-se diferença estatisticamente significativa, sendo que o G1 apresentou um nível intelectual inferior ao G2.

O projeto foi apreciado e aprovado pelas Comissões de Ética do hospital escola e das Secretarias Municipais do Bem Estar Social e da Educacão de Ribeirão Preto. Após a explicação relativa aos objetivos do trabalho e o tipo de participação requerida, foi solicitado aos pais que assinassem o Termo de Consentimento, autorizando a participação das crianças. Após essa etapa as crianças foram convidadas a participarem de forma voluntária.

\section{Instrumentos}

Foram utilizados:

- Matrizes Progressivas Coloridas de Raven - Escala Especial, avaliação do nível intelectual (normas brasileiras de Angelini, Alves, Custódio \& Duarte, 1987)

- Teste de Desempenho Escolar - TDE, avaliação objetiva do nível de desempenho escolar (normas brasileiras de Stein, 1994)

- Questionário para Caracterização do Desempenho e do Comportamento da Criança no Ambiente Escolar - DCCE (Machado, Figueiredo \& Selegato, 1989). Trata-se de um questionário apresentado sob a forma de escala de sete pontos, composta por adjetivos bipolares, englobando categorias de comportamento da criança em sala de aula em três áreas distintas: comportamento em relação à professora, aos colegas e à tarefa.

- Roteiro de Avaliação de Auto-eficácia (Anexo A): focaliza a percepção da criança quanto ao seu desempenho acadêmico e sua capacidade de realização. Trata-se de um procedimento elaborado pela segunda autora a partir dos estudos de Bandura (1993) e Shunck (1995) como parte das atividades no projeto integrado conjunto com 
a terceira e quarta autora: "Problemas associados a dificuldades escolares - construção e aplicação de procedimentos avaliativos com enfoque desenvolvimentista" (Medeiros \& Loureiro, 1999). O roteiro é composto por 20 afirmativas, dessas 12 são relacionadas à percepção da capacidade quanto ao desempenho acadêmico, compreendendo seis afirmativas com significado positivo (itens 01, 03, 05, 07, 09, 20) seis afirmativas com significado negativo (itens 02,12 , 14, 16, 18, 19); e oito afirmativas relacionadas à percepção de desempenho acadêmico, tendo como referência a avaliação de outros ou a comparação com os pares, compreendendo quatro afirmativas com significado positivo (itens $11,13,15,17$ ) e quatro afirmativas com significado negativo (itens $04,06,08,10)$. O roteiro foi testado em estudo preliminar com 20 crianças (idade entre 8 e 10 anos). O índice de estabilidade (teste e reteste) alcançado em duas avaliações, com 28 dias de intervalo foi de $92 \%$ (concordância bruta).

- Escala Comportamental Infantil A2 de Rutter - (ECI) avaliação de problemas comportamentais, preenchida pelos pais - padronizada para escolares de Ribeirão Preto (Graminha, 1994b). A ECI inclui 36 itens, fornecendo um escore global, assim como escores parciais relativos a problemas de saúde (oito), hábitos (sete) e comportamentos (21).

\section{Procedimentos}

As crianças foram avaliadas individualmente, as do G1 no ambulatório e as do G2 no CAIC. Na primeira sessão procedeu-se à aplicação do Raven. Em uma segunda sessão, procedeu-se à aplicação do TDE conforme recomendações técnicas. Com base no dados destas duas avaliações e a avaliação dos professores, os sujeitos foram incluídos nos grupos G1 (crianças com queixa de dificuldade de aprendizagem) e G2 (crianças com bom desempenho acadêmico). Em uma terceira sessão procedeu-se à aplicação do Roteiro de Avaliação de Auto-eficácia, subseqüente a uma situação semiestruturada de contato com materiais e atividades acadêmicas, visando a fornecer uma contextualização de aprendizagem antecedendo imediatamente à aplicação.

O Roteiro foi aplicado nos dez minutos finais da sessão. Os itens do Roteiro foram apresentados atravé de fita cassete gravada, evitando assim variações de entonações e pausas na leitura dos mesmos. As crianças podiam ouvir os itens tantas vezes quantas sentissem necessidade, sempre através do gravador. A examinadora, presente na situação, apenas assinalava a opção da criança na folha de respostas.
Com relação aos pais, o procedimento de aplicação da ECI teve peculiaridades em cada um dos grupos. Quanto ao G1, após uma situação de entrevista face a face, visando a coleta de informações de dados de identificação, os pais preenchiam a ECI por escrito sob a forma auto-aplicativa, própria da adaptação do instrumento. Quanto ao G2, os pais receberam em suas casas um questionário de dados de identificação e a ECI, respondendo ambos por escrito.

Quanto aos professores, estes foram contatados nas escolas de origem das crianças e responderam por escrito ao Questionário para Caracterização do Desempenho e do Comportamento da Criança no Ambiente Escolar.

\section{Tratamento dos dados}

Para a classificação dos dados relativos ao senso de auto-eficácia, as respostas foram pontuadas em 0 (zero) ou 1 (um), de acordo com a alternativa escolhida. Os sujeitos dispunham de duas alternativas de respostas, "sim" ou "não". Para as respostas favoráveis a um senso de auto-eficácia positivo foi atribuído um valor 1 (um), que pode corresponder a um sim ou não dependendo do item em questão. No conjunto de 20 itens, nas dez afirmativas positivas a resposta sim é pontuada com valor 1 (um), e nas dez afirmativas negativas a resposta não é pontuada com valor 1 (um).

Ex.: Eu tenho me saído bem.......... Sim $=1$ e Não $=0$

Eu quero parar de estudar logo......Sim $=0$ e Não $=1$

A somatória do total de pontos obtidos no conjunto de 20 itens correspondeu ao escore total, tendo-se por este critério que quanto maior a pontuação mais alto é o senso de auto-eficácia da criança. Consideraram-se para a comparação dos grupos, os resultados de cada item e o escore total.

O Roteiro foi cotado de acordo com o esquema descrito. Os dados foram quantificados e os grupos comparados mediante análise estatística através do Teste não-paramétrico de Mann-Whitney para amostras independentes. Procedeu-se à análise dos 20 itens do Roteiro comparando-se os grupos e identificando-se as diferenças estatisticamente significativas com base no Teste Exato de Fisher.

Os dados da ECI foram cotados segundo as normas de Graminha (1994b). A fim de comparar os grupos quanto aos escores parciais e total, assim como nos itens específicos aplicou-se o Teste não-paramétrico de MannWhitney. Objetivando-se a comparação dos grupos através do Teste Qui-quadrado quanto ao nível de funcionamento, tomou-se a proposição de Graminha e Coelho (1994) relativa à nota de ponto de corte (escore 
total >16) como indicativa da necessidade de encaminhamentos para atendimento psicológico.

Para determinar a existência de possíveis associações entre a avaliação do senso de auto-eficácia realizada pelas crianças, o nível intelectual, e o funcionamento comportamental informado pelos pais através da ECI, procedeu-se à aplicação da Matriz de correlação nãoparamétrica de Spearman.

\section{Resultados}

Primeiramente serão apresentados os dados da avaliação do senso de auto-eficácia com relação ao escore total e a discriminação dos itens. Em seguida, serão apresentados os dados relativos à ECI, destacando-se os escores parciais e total e os itens com diferenças estatisticamente significativas e, por fim, a associação entre os dados dos dois instrumentos em questão. Os dados

Tabela 1. Auto-eficácia - Média, Desvio Padrão e Mediana da Comparação do Escore Total dos Grupos Avaliados (Teste não-paramétrico de Mann-Whitney)

\begin{tabular}{ccccc}
\hline Grupos & Média & $d p$ & Mediana & $p$ \\
\hline G1 & 12,19 & 4,22 & 12,50 & 0,001 \\
G2 & 18,54 & 2,16 & 19,00 & \\
\hline
\end{tabular}

relativos ao Roteiro de Avaliação de Auto-eficácia quanto à comparação do escore total de cada grupo estão apresentados na Tabela 1

Observa-se diferença altamente significativa entre os dois grupos quando da avaliação do escore total, considerando-se $p<0,001$. O G1 obteve pontuação menor que o $\mathrm{G} 2$, caracterizando uma avaliação mais baixa do senso de auto-eficácia por parte das crianças com queixa de dificuldade de aprendizagem. A Tabela 2 apresenta os dados do Roteiro de Avaliação de Autoeficácia discriminando os itens com diferenças estatisticamente significativas

Pode-se observar que, dentre os 20 itens apresentados, 12 itens diferenciam os grupos com significância estatística. Observa-se que destes 12 itens, três têm valoração positiva em relação ao senso de auto-eficácia e nove têm uma valoração negativa. Nos itens com valoração positiva que diferenciam os grupos com significância estatística podese observar que o G2 apresenta uma porcentagem superior ao G1, indicando que as crianças com bom desempenho apresentaram um senso de auto-eficácia mais alto quando comparadas com o G1. Em contrapartida, nos itens com valoração negativa, o G1 apresenta uma porcentagem superior ao $G 2$, indicando um senso de auto-eficácia mais baixo para os sujeitos do G1.

Observa-se ainda que oito itens não apresentaram diferenças estatisticamente significativas e que destes, sete

Tabela 2. Auto-eficácia - Itens com Diferenças Estatisticamente Significativas, na Comparação da Porcentagem de Sujeitos dos Dois Grupos, Discriminando a Valoração Positiva e Negativa

\begin{tabular}{clccc}
\hline & Itens & $\mathrm{G} 1$ & $\mathrm{G} 2$ & $p$ \\
\hline+ & 1.Eu tenho me saído bem & 73,1 & 96,2 & 0,05 \\
+ & 13.Eu acabo as atividades no mesmo tempo que os meus colegas. & 61,5 & 92,3 & 0,01 \\
+ & 15.Minha professora me considera um bom aluno. & 73,1 & 96,2 & 0,05 \\
- & 4.Minha família me considera um aluno fraco & 42,3 & 11,5 & 0,05 \\
- & 6.Minha professora me considera um aluno fraco & 38,5 & 7,7 & 0,05 \\
- & 8.Eu demoro mais que os outros para acabar as atividades. & 38,5 & 7,7 & 0,05 \\
- & 10.Eu tenho mais dificuldade para aprender que os meus colegas. & 76,9 & 15,4 & 0,001 \\
- & 12.Eu esqueço rápido o que aprendi. & 50 & 0 & 0,001 \\
- & 14.Eu tenho dificuldade para escrever as palavras que são ditadas. & 61,5 & 7,7 & 0,001 \\
- & 16.Eu tenho dificuldade para fazer cópia. & 46,2 & 7,7 & 0,01 \\
- & 18.Eu tenho dificuldade para ler. & 61,5 & 3,8 & 0,001 \\
- & 19.Eu tenho me saído mal. & 50 & 7,7 & 0,01 \\
+ & 3.Eu consigo ler com facilidade. & 76,9 & 92,3 & $\mathrm{~ns}$ \\
+ & 5.Eu consigo copiar com facilidade. & 73,1 & 92,3 & $\mathrm{~ns}$ \\
+ & 7.Eu consigo escrever as palavras que são ditadas. & 76,9 & 96,2 & $\mathrm{~ns}$ \\
+ & 9.Eu lembro com facilidade do que aprendi. & 76,9 & 88,5 & $\mathrm{~ns}$ \\
+ & 11.Eu aprendo tão bem quanto os meus colegas. & 65,4 & 80,8 & $\mathrm{~ns}$ \\
+ & 17.Minha família me considera bom aluno. & 88,5 & 96,2 & $\mathrm{~ns}$ \\
+ & 20.Eu quero continuar estudando por muitos anos. & 88,5 & 100 & $\mathrm{~ns}$ \\
- & 2.Eu quero parar de estudar logo. & 19,2 & 3,8 & $\mathrm{~ns}$ \\
\hline
\end{tabular}

Psicologia: Reflexão e Crítica, 2000, 13(3), pp.327-336 
332 Paula Cristina Medeiros, Sonia Regina Loureiro, Maria Beatrir Martins Linhares \& Edna Maria Marturano

itens são com valoração positiva, onde o G2 novamente apresentou uma porcentagem superior ao G1. A Tabela 3 apresenta os dados obtidos da ECI respondida pelos pais das crianças do G1 e do G2, comparando os escores parciais e total.

Observa-se que os grupos se diferenciam significativamente com relação à área de comportamento e ao escore total. Quanto ao escore parcial relativo à área de comportamento o G1 apresenta média maior do que o G2, indicando maiores dificuldades nesta área. Quanto ao escore total, incluindo todos os itens, o G1 apresenta também média significativamente maior do que o G2.

Quanto ao escore total superior à nota de corte - 16 pontos (Graminha \& Coelho, 1994), observa-se que $77 \%$ dos sujeitos do G1 apresentaram valores de escore total acima de 16 pontos, sugerindo maior necessidade de atendimento psicológico. A Tabela 4 apresenta a comparação dos grupos, discriminando os itens da ECI com diferenças estatisticamente significativas.
Observa-se que sete itens diferenciam significativamente os grupos. Nos dois primeiros itens relativos à área de saúde, o G1 apresenta uma média inferior quando comparado com G2. Na área de hábitos apenas o item referente à dificuldade de fala diferencia os grupos. $\mathrm{O}$ G1 apresenta maior número de sujeitos com esta dificuldade e nenhuma criança do G2 foi referida como tendo-a. Nos quatro itens referentes à área de comportamento, o G1 novamente apresenta um número maior de sujeitos com dificuldade na comparação com G2.

A correlação entre as variáveis senso de auto-eficácia, funcionamento comportamental, e nível intelectual das crianças, através da Matriz de Correlação NãoParamétrica de Spearman, é apresentada na Tabela 5.

Com relação ao senso de auto-eficácia, observa-se correlação com o escore parcial da área de comportamento e o escore total da ECI. Analisando-se a direção das relações pode-se dizer que as crianças que

Tabela 3. Escala Comportamental Infantil A2 Rutter (ECI) - Comparação dos Escores Parciais e Total dos Grupos Avaliados (Teste não-paramétrico de Mann-Whitney)

\begin{tabular}{lcrrrr}
\hline Áreas & Grupos & Média & $\not p$ & Mediana & \multicolumn{1}{c}{$p$} \\
\hline Saúde & 1 & 2,77 & 1,56 & 3,04 & \\
& 2 & 2,88 & 2,03 & 2,50 & \\
Hábitos & 1 & 2,62 & 1,94 & 3,00 & \\
& 2 & 1,88 & 1,37 & 2,00 & \\
Comport. & 1 & 15,23 & 6,00 & 15,50 & 0,05 \\
& 2 & 11,00 & 7,03 & 9,50 & \\
Escore Total & 1 & 20,62 & 7,46 & 21,00 & 0,05 \\
& 2 & 15,77 & 8,30 & 14,00 &
\end{tabular}

Tabela 4. Escala Comportamental Infantil A2 Rutter (ECI) - Comparação dos Grupos quanto aos Itens com Diferenças Estatisticamente Significativas

\begin{tabular}{|c|c|c|c|c|c|c|}
\hline Área & Itens & Grupo & Média & $d p$ & Mediana & $p$ \\
\hline \multirow[t]{4}{*}{ Saúde } & \multirow[t]{2}{*}{ Faz xixi na cama ou nas calças } & 1 & 0,00 & 0,00 & 0,00 & \multirow[t]{2}{*}{0,05} \\
\hline & & 2 & 0,19 & 0,49 & 0,00 & \\
\hline & \multirow[t]{2}{*}{ "Mata" ou "enforca" aula } & 1 & 0,00 & 0,00 & 0,00 & \multirow[t]{2}{*}{0,05} \\
\hline & & 2 & 0,19 & 0,40 & 0,00 & \\
\hline \multirow[t]{2}{*}{ Hábitos } & \multirow{2}{*}{$\begin{array}{l}\text { Há alguma dificuldade com a fala além } \\
\text { da gagueira. }\end{array}$} & 1 & 0,62 & 0,80 & 0,00 & \multirow[t]{2}{*}{0,01} \\
\hline & & 2 & 0,00 & 0,00 & 0,00 & \\
\hline \multirow[t]{8}{*}{ Comportamento } & \multirow{2}{*}{$\begin{array}{l}\text { Fica facilmente preocupado, } \\
\text { preocupa-se com tudo. }\end{array}$} & 1 & 1,04 & 0,87 & 1,00 & \multirow[t]{2}{*}{0,05} \\
\hline & & 2 & 0,50 & 0,76 & 0,00 & \\
\hline & Tende a ser uma criança fechada - um & 1 & 0,77 & 0,86 & 0,50 & \multirow[t]{2}{*}{0,05} \\
\hline & tanto solitária & 2 & 0,31 & 0,68 & 0,00 & \\
\hline & Irritável, & 1 & 1,19 & 0,85 & 1,00 & \multirow[t]{2}{*}{0,05} \\
\hline & perde as "estribeiras" & 2 & 0,73 & 0,72 & 1,00 & \\
\hline & É uma criança insegura, não tem & 1 & 1,00 & 0,75 & 1,00 & \multirow[t]{2}{*}{0,01} \\
\hline & confiança em si mesma & 2 & 0,27 & 0,53 & 0,00 & \\
\hline
\end{tabular}

(Teste não-paramétrico de Mann-Whitney) 
Tabela 5. Associações entre Avaliação do Senso de Auto-eficácia, Funcionamento Comportamental, e o Nível Intelectual das Crianças (Matriz de correlação não-paramétrica de Spearman)

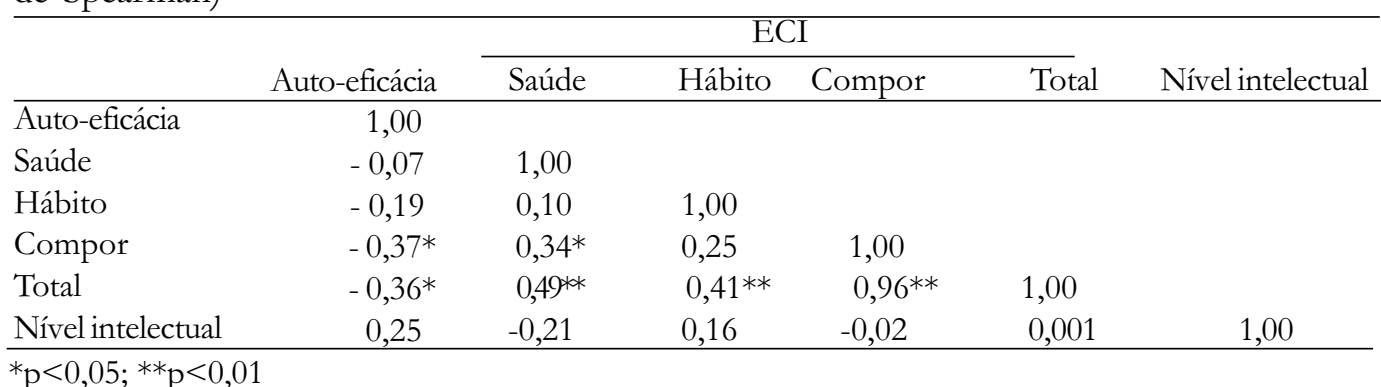

apresentam pontuação mais alta no sentido de problemas comportamentais apresentam pontuação mais baixa no Roteiro de Avaliação de Auto-eficácia, sugerindo baixo senso de auto-eficácia.

Verificam-se correlações entre os escores parciais das áreas de saúde, hábito e de comportamento e o escore total da ECI. Observa-se também correlação entre o escore das áreas de saúde e comportamento. Não se observa correlação entre o nível intelectual, o senso de auto-eficácia e o nível de funcionamento comportamental das crianças.

\section{Discussão}

Retomando o objetivo do presente trabalho, podese observar que os dois grupos estudados apresentaram diferenças importantes no que se refere ao senso de autoeficácia e manifestações comportamentais. O grupo de crianças com queixa de dificuldade de aprendizagem apresenta um senso de auto-eficácia mais baixo em relação às crianças com bom desempenho, considerando-se pouco competente para a execução com sucesso de determinadas atividades acadêmicas. Este padrão de funcionamento é concordante com dados de outros estudos que apontam as relações de baixo senso de autoeficácia com baixo desempenho acadêmico (Bandura e cols., 1996; Pajares, 1996; Pajares \& Valiante, 1997; Schunk, 1995). Pintrich e colaboradores (1994) compararam crianças com dificuldade de aprendizagem e sem dificuldade, investigando as diferenças nas crenças motivacionais e o conhecimento metacognitivo dos estudantes. Os resultados indicaram que estudantes que atribuíam seus sucessos na leitura a causas internas, eram menos ansiosos, apresentavam uma orientação mais focalizada, apresentavam um senso de auto-eficácia mais alto, eram mais metacognitivos e tinham um desempenho acadêmico melhor.

Psicologia: Reflexão e Crítica, 2000, 13(3), pp.327-336
Quanto à análise dos dados do Roteiro de Avaliação de Auto-eficácia, é interessante destacar que nove, dos 12 itens com diferença significativa, se relacionam com afirmativas com sentido negativo em relação ao senso de auto-eficácia. Na maioria das vezes, estas afirmativas iniciam a frase retratando a dificuldade: "eu demoro, en esqueç, eu tenho mais dificuldade". Pode-se pensar que essas afirmativas, com sentido negativo, tenham um impacto maior para as crianças com dificuldade de aprendizagem, possivelmente por serem parte da sua experiência cotidiana em comentários ou avaliações dos pais, das professoras ou dos colegas.

Observa-se ainda que os itens com sentido negativo, que apresentaram diferença significativa, referem-se à percepções baseadas em julgamentos dos outros ou comparações com os colegas. Destaca-se aqui a importância do meio ambiente enquanto fonte de influência para a formação das crenças das crianças. De acordo com Bandura (1989a) as comparações sociais, a avaliação do desempenho dos colegas e avaliação que as pessoas recebem dos outros, têm forte influência sobre o desenvolvimento do senso de auto-eficácia. Estas percepções baseadas no julgamento das outras pessoas estão relacionadas ao senso de auto-eficácia social. Um alto senso de auto-eficácia social pode promover satisfação e sustentar relacionamentos sociais positivos.

Com relação aos aspectos comportamentais, avaliados através da ECI, de forma geral observa-se que os pais das crianças encaminhadas com queixa de dificuldade de aprendizagem, tendem a percebê-las com mais problemas, se comparadas ao grupo com bom desempenho. Quando se analisa as áreas separadamente, observa-se um dado interessante quanto à área de saúde. Nota-se que os sujeitos do grupo com bom desempenho apresentam mais dificuldades nos itens matar aula e fazer xixi na cama, quando comparado com o grupo com dificuldade de aprendizagem. Pode-se pensar que embora estas crianças tenham bom desempenho acadêmico, 
podem estar suscetíveis a outras dificuldades. Loureiro, Marturano, Linhares, Machado e Silva (1994), estudando três grupos de crianças (com história de atraso escolar e procura de atendimento psicológico, com história de atraso sem procura por atendimento e sem atraso escolar) verificaram manifestações conflituosas nesses grupos. As crianças sem atraso escolar apresentaram indícios sugestivos de manifestações de controle sobre o meio e dissimulação dos conflitos. Por outro lado, pode-se pensar que a ausência do indicador "matar aula" para as crianças do G1 tenha relação com a preocupação dos pais destas crianças quanto às atividades acadêmicas, fiscalizando mais a freqüência e assiduidade de seus filhos às aulas.

Os problemas da área de comportamento apresentados pelas crianças com queixa de dificuldade de aprendizagem referem-se em geral a aspectos internos como preocupação, fechamento ou solidão, irritabilidade e insegurança. Estudos têm sugerido a presença de manifestações emocionais e comportamentais desadaptadas quando da associação dificuldades de aprendizagem e fatores afetivos (Graminha, 1994a). Marturano, Loureiro, Linhares e Machado (1997) encontraram que crianças com atraso escolar, identificadas pelas famílias que buscaram assistência psicológica, denotaram um empobrecimento na sua capacidade produtiva e comprometimentos associados à estruturação da personalidade, bem como uma auto-imagem negativa com intensos sentimentos de inferioridade e menos valia.

As pesquisas que relacionam auto-eficácia e afeto são concordantes ao apontarem uma relação negativa entre auto-eficácia e ansiedade. Pintrich e colaboradores (1994), relatam que alto nível de ansiedade aparece relacionado com mau desempenho em tarefas de leitura, sendo que os estudantes que sentiam-se muito preocupados quanto ao seu desempenho nos testes de leitura não se saíam bem e apresentavam um baixo senso de auto-eficácia Bandura e colaboradores (1996), referem que um baixo senso de auto-eficácia para exercer controle sobre eventos estressores e a alta valorização dada aos resultados propiciam sentimentos de vazio e depressão.

Ao considerar-se a correlação entre o senso de autoeficácia e o escore parcial da área de comportamento e o escore total da ECI, observa-se que as crianças que apresentaram pontuação mais alta no sentido de problemas comportamentais apresentaram pontuação mais baixa na avaliação do senso de auto-eficácia. Podese pensar nas possíveis relações do senso de auto-eficácia sobre o comportamento, ou seja, o quanto um baixo senso de auto-eficácia pode estar associado a comportamentos pouco adaptados.
Outro dado importante a ser mencionado refere-se ao nível intelectual das crianças dos dois grupos. Apesar de os grupos se diferenciarem estatisticamente com relação ao nível intelectual, esta variável não mostrou correlação com o senso de auto-eficácia ou mesmo com problemas comportamentais. Deve-se ressaltar que apesar de o nível intelectual apresentar-se como significativamente diferente para os grupos, foi critério de inclusão que este fosse pelo menos médio inferior. Partindo-se do pressuposto de que as crianças com dificuldade de aprendizagem contavam com indício de recurso intelectual potencial, ou seja, suas dificuldades não poderiam ser atribuídas a um rebaixamento intelectual, pode-se pensar que embora com recursos estas crianças não estejam em condições de utilizá-los. Para Bandura (1993), a realização pessoal não requer só o recurso, mas sim um alto senso de auto-eficácia para usá-las da melhor forma. O rendimento escolar pode ser suscetível às flutuações do senso de auto-eficácia, e quando baixo pode levar a criança a render menos do que realmente poderia, não porque não seja capaz, mas sim porque não acredita em sua capacidade.

Segundo sugerido por Schunk (1995) e Pajares (1996), o senso de auto-eficácia influencia o desempenho acadêmico tanto por uma ação direta como através do seu impacto nos processos de motivação, auto-regulação e auto-percepção, expectativas de resultados, escolhas e interesses. Entretanto, o tipo de instrumento utilizado para avaliar o senso de auto-eficácia neste estudo, não permite uma análise aprofundada quanto ao impacto desta nos processos específicos citados. Para tal, seriam necessárias provas de desempenho associadas às variáveis específicas e um instrumento construído para estas avaliações.

A avaliação do senso de auto-eficácia no contexto acadêmico pode ser de utilidade para o planejamento de procedimentos de intervenção. Um aumento evidente no modo como os estudantes se percebem ao nível acadêmico, afeta seu desempenho e comportamentos acadêmicos subseqüentes (Pajares, 1996). Estudantes com dificuldade de aprendizagem que receberam intervenção baseada em diálogo interativo e instruções durante o processo, demonstraram um aumento substancial no senso de auto-eficácia para a escrita (Wong, Butler, Ficzere \& Kuperis, 1996). Ainda sobre processos de intervenção que visam alterar o senso de auto-eficácia frente à tarefas motoras específicas, Zimmerman e Kitsantas (1997) concluíram que o processo de auto-regulação, através do estabelecimento de metas e do auto-monitoramento, pode otimizar não só o desempenho em si, mas também o senso de auto-eficácia e interesse intrínseco pela tarefa. 
Os dados do presente trabalho, sugerem que crianças encaminhadas com queixa de dificuldade de aprendizagem apresentaram um baixo senso de autoeficácia e foram avaliadas pelos seus pais como tendo mais problemas comportamentais quando comparadas às crianças com bom desempenho escolar.

A etapa do ensino fundamental, correspondente a fase dos seis aos doze anos, segundo Erikson (1950/1971) constitui-se um período em que as aquisições de habilidades e conhecimentos têm papel decisivo para o desenvolvimento. Nesse sentido, as intervenções favorecedoras de um alto senso de auto-eficácia podem possibilitar não só um melhor nível de rendimento, como também implementar a capacidade de se envolver com situações de aprendizagem.

Como implicação dessa destaca-se a importância de se oferecer às crianças ferramentas que lhes permitam, além da aquisição de habilidades, desenvolver crenças mais positivas em relação às suas próprias capacidades de realização. Sugere-se que no trabalho com crianças na fase inicial de aprendizagem formal, mesmo quando seu rendimento está abaixo do esperado seja valorizado o desenvolvimento da auto-eficácia como um recurso favorecedor do processo de aprendizagem.

\section{Referências}

Angelini, A., Alves, I., Custódio, E. \& Duarte, W. (1987). Manual das matrizes progressivas coloridas Raven. São Paulo: Casa do Psicólogo.

Bandura, A. (1989a). Human agency in social cognitive theory. America Psychologist, 44, 1175-1184.

Bandura, A. (1989b). Regulation of cognitive processes through perceived self-efficacy. Developmental Psychology, 25, 729-735. Bandura, A. (1993). Perceived self-efficacy in cognitive development and
functioning. Educational Psychologist, 28, 117-148.

Bandura, A., Barbaranelli, C., Caprara, G. V. \& Pastorelli, C. (1996). Multifaceted impact of self-efficacy beliefs on academic functioning. Cbild Development, 67, 1206-1222.

Bandura, A. \& Schunk, D. H. (1981). Cultivating competence, self-efficacy, and intrinsic interest through proximal self-motivation. Journal of Personality and Social Psychology, 41, 586-598.

Boruchovitch, E. (1994). As variáveis psicológicas e o processode aprendizagem: Uma contribuição para a psicologia escolar. Psicologia: Teoria e Pesquisa, 10, 129-139.

Chapman, J. W., Cullen, J. L., Boersma, F. J. \& Maguire, T. O. (1981). Affective variables and school achievement: A study of possible causal influences. Canada Journal of Bebavior Science, 3, 181-192.

Erikson, E. (1971). Infancia e sociedade (G. Amado, Trad.) Rio de Janeiro: Zahar. (Original publicado em 1950)

Graminha, S. S. V. (1994a). Problemas emocionais/comportamentais em uma amostra de escolares: Incidência em função do sexo e idade. Psico, 25, 49-74.

Graminha, S. S. V. (1994b). A escala comportamental infantil de Rutter A2: Estudos de adaptação e fidedignidade. Estudos de Psicologia, 11, 34-42.

Graminha, S. S. V. \& Coelho, W. F. (1994). Problemas emocionais/comportamentais em crianças que necessitam ou não de atendimento psicológico ou psiquiátrico [Resumo]. Em Sociedade Brasileira de Psicologia (Org.), Resumos da XXIV Reunião Anual de Psicologia (p. 263). Ribeirão Preto: SBP.
Lindahl, N. Z. (1988). Personalidade humana e cultura: Aplicações educacionais da teoria de Erik Erikson. Revista Brasileira de Estudos Pedagogicos, $69,492-509$

Linhares, M. B. M. (1998). Atendimento psicopedagógico de crianças em serviço especializado de psicologia infantil na área de saúde: Uma perspectiva desenvolvimentista. Psicopedagogia, 17, 30-36.

Linhares, M. B. M., Parreira, V. L. C., Maturano, A. C. \& Sant'Anna, S. C. (1993). Caracterização dos motivos da procura de atendimento infantil em um serviço de psicopedagogia clínica. Medicina, 26, 148-160.

Loureiro, S. R., Marturano, E. M., Linhares, M. B. M., Machado, V. L. S. \& Silva, S. R. (1994). Crianças com queixa de dificuldade escolar: Avaliação psicológica através de técnica gráfica. Arquivos Brasileiros de Psicologia Aplicada, 46, 161-182.

Machado, V. L. S., Figueiredo, M. A. \& Selegato, M. V. (1989). Caracterização do comportamento de alunos em sala de aula através de escalas de desempenho. Estudos de Psicologia, 6, 50-76.

Martín, E. \& Marchesi, A. (1995). Da terminologia do distúrbio às necessidades educacionais especiais. Em C. Coll, J. Palácios \& A. Marchesi (Orgs.), Desenvolvimento psicologico e educacáa: Necessidades educativas especiais e aprendizagem escolar (pp. 7-35) (M. A. G. Domingues, Trad.). Porto Alegre: Artes Médicas.

Marturano, E. M. (1997). A criança, o insucesso escolar precoce e a família: Condições de resiliência e vulnerabilidade. Em E. M. Marturano, S R. Loureiro \& A. W. Zuardi (Orgs.), Estudos em saúde mental (pp. 132 145). Ribeirão Preto: Comissão de Pós-Graduação em Saúde Mental da FMRP/USP.

Marturano, E. M., Linhares, M. B. M. \& Parreira, V. L. C. (1993). Problemas emocionais e comportamentais associados a dificuldades na aprendizagem escolar. Medicina, 26, 161-175.

Marturano, E. M., Loureiro, S. R., Linhares, M. B. M. \& Machado, V. L. S (1997). A avaliação psicológica pode fornecer indicadores de problemas associados a dificuldades escolares? Em E. M. Marturano, 47). Ribeirão Preto: Comissão de Pós-Graduação em Saúde Mental da
4. 47). Ribeirão

Medeiros \& Loureiro, S. R. (1999). Auto-eficácia e aspectos comportamentais de criancas com dificuldade de aprendizagem. [Resumo] Em Sociedade Brasileira de Psicologia (Org.), Programas e Resumos da XXIX Reunião Anua de Psicologia, (p. 152). Ribeirão Preto: SBP

Pajares, F. (1996). Self-efficacy beliefs in academic settings. Review of Educational Research, 66, 543-578.

Pajares, F. \& Valiante, G. (1997). The predictive and mediational role of the writing self-efficacy beliefs of upper elementary students. Journal of Educational Research, 90, 353-360.

Pintrich， P. R., Anderman, E. M. \& Klobucar, C. (1994). Intraindividual differences in motivation and cognition in students with and without learning disabilities. Journal of Learning Disabilities, 27, 360-370.

Schunk, D. H. (1995). Self-efficacy and education and instruction. Em J. E. Maddux (Org.), Self-efficacy, adaptation, and adjustment: Theory, researcb, and Maddux (Org.), Self-efficacy, adaptation, and adjustment:
application (pp. 281-301). New York: Plenum Press.

Stein, L. M.(1994). TDE - Teste de desempenho escolar: Manual para aplicacãa e in terpretacáão. São Paulo: Casa do Psicólogo.

Wong, B. Y. L., Butler, D. L., Ficzere, S. A. \& Kuperris, S. (1996). Teaching low achievers and students with learning disabilities to plan, write, and revise opinion essays. Journal of Learning Disabilities, 29, 197-212.

Zimmerman, B. J. \& Kitsantas, A. (1997). Developmental phases in selfregulation: Shifting from process goals to outcome goals. Journal of Educational Psychology, 89, 29-36. 
336 Paula Cristina Medeiros, Sonia Regina Loureiro, Maria Beatriz Martins Linhares \& Edna Maria Marturano

Anexo A

Roteiro de Avaliação de Auto-Eficácia

Vou apresentar para você algumas frases e você vai me dizer o quanto elas tem a ver com o seu jeito de ser. Basta me responder SIM quando elas se parecerem e NÃO quando elas forem diferentes do seu jeito.

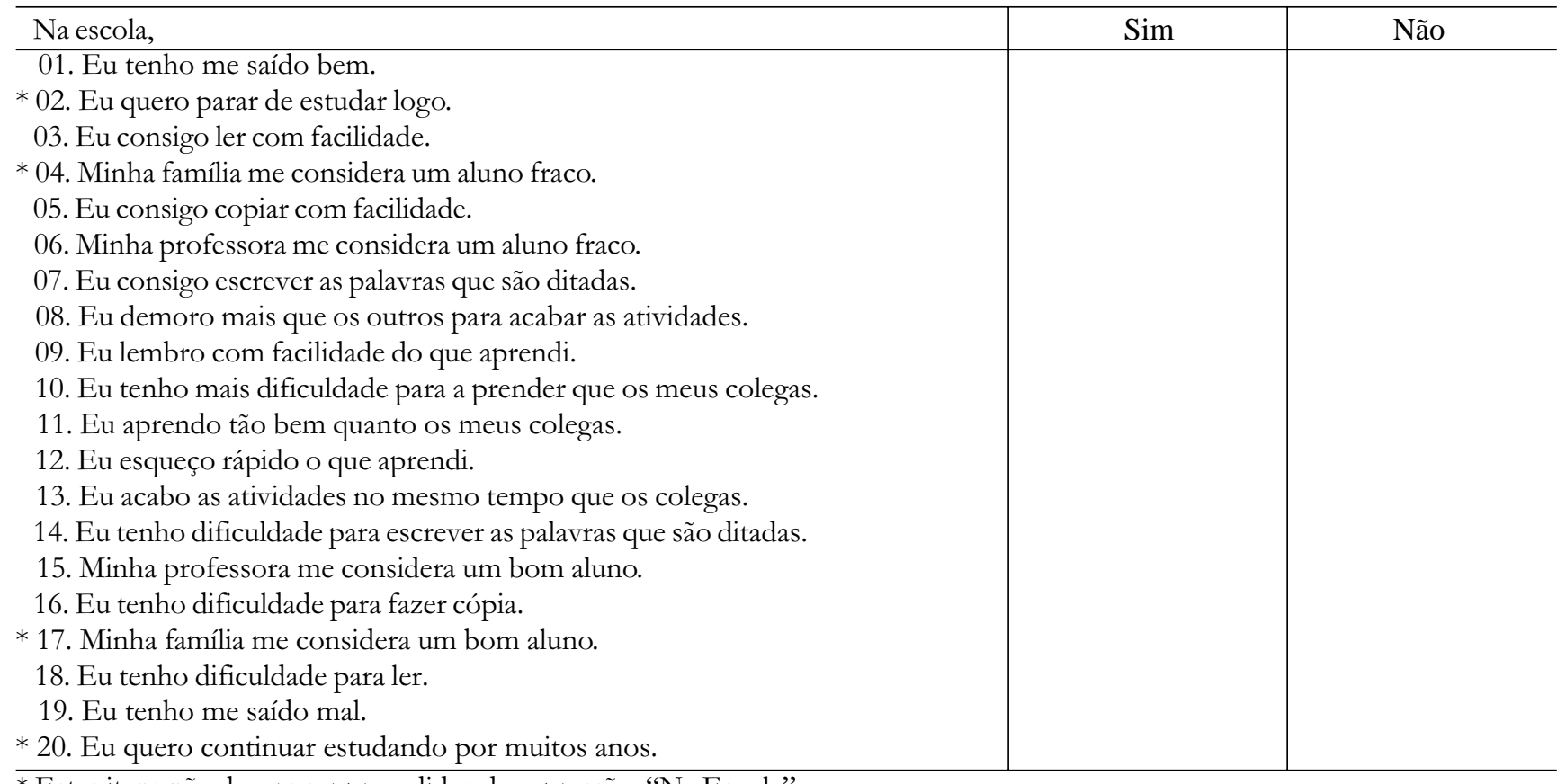

* Estes itens não devem ser precedidos da expressão: "Na Escola".

Sobre as autoras:

Paula Cristina Medeiros é Psicóloga, Especialista em Problemas de Aprendizagem e Psicopedagogia,

Mestranda em Saúde Mental pela Faculdade de Medicina de Ribeirão Preto, Universidade de São Paulo.

Sonia Regina Loureiro é Psicóloga, Professora Doutora da Faculdade de Medicina de Ribeirão Preto/USP e docente nos Cursos de Pós-Graduação em Ciências Médicas (área Saúde Mental) e Psicologia, na Universidade de São Paulo/Ribeirão Preto. Coordena o Serviço de Psicodiagnóstico do Hospital de Clínicas de Ribeirão Preto, onde mantém atividades de formação de recursos humanos e de pesquisa com instrumentos e procedimentos de avaliação em diferentes contextos psicossociais. Maria Beatriz Martins Linhares é Psicóloga, Especialista em Psicologia Clínica Infantil, Mestre em Educação Especial, Doutora em Psicologia Experimental, Docente da Faculdade de Medicina de Ribeirão Preto, Universidade de São Paulo, Orientadora nos Cursos de Pós-Graduação em Saúde Mental (FMRP/USP) e Psicologia (FFCLRP/USP).

Edna Maria Marturano é Psicóloga, Professora Titular da Faculdade de Medicina de Ribeirão Preto/USP e Docente nos Cursos de Pós-Graduação em Ciências Médicas (área Saúde Mental) e Psicologia, na Universidade de São Paulo/Ribeirão Preto. Coordena o Ambulatório de Psicologia Infantil do Hospital de Clínicas de Ribeirão Preto, onde mantém atividades de pesquisa, formação de recursos humanos e desenvolvimento de intervenções preventivas, focalizando crianças que vivem em situação de risco psicossocial e suas famílias. 